\title{
Simulation analysis of RED with short lived TCP connections
}

\author{
Eitan Altman ${ }^{\mathrm{a}, *}$, Tania Jiménez ${ }^{\mathrm{b}}$ \\ a INRIA, BP93, 2004 Route des Lucioles, 06902 Sophia Antipolis Cedex, France \\ b CESIMO, Facultad de Ingeniera, Univ. de Los Andes, 5101 Mérida, Venezuela
}

Received 5 October 2003; received in revised form 6 October 2003; accepted 8 October 2003

Responsible Editor: S. Low

\begin{abstract}
Several objectives have been identified in developing the random early drop (RED): decreasing queueing delay, increasing throughput, and increasing fairness between short and long lived connections. It has been believed that indeed the drop probability of a packet in RED does not depend on the size of the file to which it belongs. In this paper we study the fairness properties of RED where fairness is taken with respect to the size of the transferred file. We focus on short lived TCP sessions. Our findings are that (i) in terms of loss probabilities, RED is unfair: it favors short sessions, (ii) RED is fairer in terms of the average throughput of a session (as a function of its size) than in terms of loss probabilities. We study various loading regimes, with various versions of RED.
\end{abstract}

(C) 2003 Elsevier B.V. All rights reserved.

Keywords: RED; TCP; Buffer management; Simulations; Fairness

\section{Introduction}

One of the objectives of random early drop (RED) has been to get rid of the bias of drop-tail buffers against bursty traffic [3]. It has been confirmed in [4, Section IX] that indeed RED gets rid of this bias and is fair in terms of fraction of lost packets. This has been demonstrated in a framework where both bursty as well as "smooth" traffic were taken as permanent FTP connections. The higher burstiness was obtained by using smaller windows and longer round-trip times. In a recent paper [7], the authors show that conclusions drawn

\footnotetext{
${ }^{*}$ Corresponding author. Tel.: +33-4-92-38-77-86.

E-mail address: eitan.altman@sophia.inria.fr (E. Altman).
}

from simulating permanent TCP connections can be qualitatively quite different than those obtained from simulations of transfers that have large time scale variability. The latter is obtained by replacing the infinite source model by ones in which transfered files have heavy-tailed distributions. This motivated us to question the qualitative conclusions drawn in [4] and restudy the fairness issue using Pareto distributed file sizes. We are interested in the following questions: (i) how does the drop probability of a packet depend on the file size? (ii) how does the average throughput experienced by a file depend on its size?

If we identify arrival of packets from a file as a "batch" (which is justified by our simulations), then the fairness in terms of the file size also answer the question of fairness in terms of batch sizes. 
Our main findings are that in terms of loss probabilities, RED is biased against long files. Furthermore, we show that this bias increases as the workload decreases. We study this phenomenon and provide an explanation for it. We further show that RED is fairer in terms of throughput than it is in terms of loss probabilities. When comparing to drop-tail buffers, we see that for all loads, RED has smaller loss probabilities for almost all transfer sizes; the drop-tail buffer is more fair in terms of drop probabilities (as a function of the transfer size) since it has many more drops for short file transfers. In terms of throughput, RED is slightly fairer than the drop-tail buffer. Our conclusions similar for four different variants of RED that we tested.

We briefly mention some related work. Fair RED (FRED) is proposed in [10] which needs however to keep some per-active-flow states. ${ }^{1}$ Its performance is analyzed by simulating permanent TCP connections. In [14], stabilized RED (SRED) is proposed, requiring per-flow state information too. Unlike RED, the drop probabilities depend only on the instantaneous buffer occupation and on the estimated number of active flows. Fairness according to the transfer size is not considered there. [2] analyzes biasness with respect to bursty traffic. Both smooth as well as bursty traffic are modeled as Poisson processes, but in the smooth case each arrival corresponds to a single packet, where as in the bursty traffic case each arrival brings a batch of $B$ packets. The conclusions of the paper are that indeed RED decreases the bias against bursty traffic. The traffic models used are not closed loop (they do not adapt to congestion), and as we learn from [1] and [7, Section 4], qualitative behavior of closed loop traffic can be quite different from open loop. Although there are also some TCP simulations of RED in [2], these use permanent connections. Other papers studied RED using long lived TCP connections $[6,9,11-13,15-$ $17]$ as well as short lived connections $[8,17]$. But the fairness issue is not examined in these references.

\footnotetext{
${ }^{1}$ It is argued in [10] that dropping "fairly" packets does not guarantee fair bandwidth sharing. Interestingly, our simulations show that bandwidth sharing is fairer in RED than could be expected because RED is unfair in dropping packets.
}

We study the standard as well as the adaptive RED version, both with and without the gentle option of RED. ${ }^{2}$ We introduce the model and simulation setup in Section 2, present some preliminary simulation results in Section 3 , then present the fairness results in terms of the loss probabilities in Section 4 and in terms of the throughput in Section 5. The analysis of these results and explanation of the causes for the observed behavior are given in Section 6, and we end with a concluding section.

\section{Model and simulation setup}

\subsection{Traffic model and topology}

There are $X$ source nodes ( $X$ is determined below) connected to a bottleneck link $N$ through which the packets are forwarded to a common destination $D$. We consider transfer of files from each source node whose distribution is Pareto with an average size of 30 kbytes and with shape parameter of $1.1{ }^{3}$ From each node, the time between beginning of transmissions of files has an exponential distribution with average of $0.9 \mathrm{~s}$. Thus a source can be sending simultaneously packets belonging to more than one connection. The bottleneck link $N-D$ has a delay of $1 \mathrm{~ms}$ and a queue of size 50 packets. The rate of the link is $1.8 \mathrm{Mbps}$ (it will later be increased up to $2.5 \mathrm{Mbps}$ in order to study the dependence of the performance in the load of the system). The other links, i.e. between the sources and $N$, have all $100 \mathrm{Mbps}$ bandwidth and a delay of $1 \mathrm{~ms}$. The network is depicted in Fig. 1. The average input rate of data information into the bottleneck link from each node is $30 \times 10^{3} \times 8 / 0.9 \mathrm{~s}=266.67 \mathrm{kbps}$. We took packet sizes of 500 bytes, so with an additional overhead of 40 bytes, their size is 540 bytes. Taking this into account, the total transmission rate of bits (without counting the retransmissions) from each source is $288 \mathrm{kbps}$. Thus the system can

\footnotetext{
${ }^{2}$ http://www.icir.org/floyd/red/gentle.html.

${ }^{3}$ Recall that for Pareto distribution, $\operatorname{Pr}($ size $>s)=(k / s)^{\beta}$ and $E[$ size $]=\beta k /(\beta-1)$ where $\beta$ is the shape parameter; thus the parameter $k$ equals to 2727.27.
} 


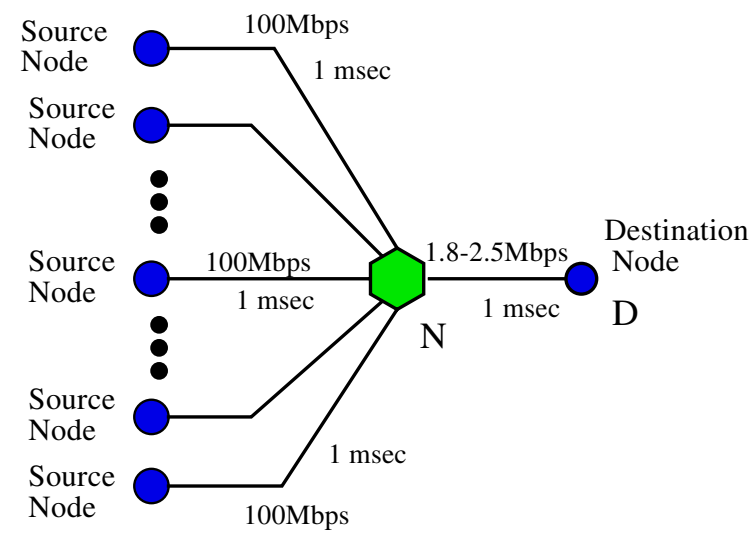

Fig. 1. Network topology.

support at most $X=6$ input nodes in order that the number of sessions does not grow to infinity. The total arrival rate of bits (without retransmissions) is then $1.728 \mathrm{Mbps}$, so we work in a heavy load regime close to 1 , i.e. $\rho=1.728 / 1.8=0.96$. Increasing the bottleneck capacity to $2.5 \mathrm{Mbps}$ will allow us to check the light load regime where $\rho=1.728 / 2.5=0.6912$. Our simulations lasted $2500 \mathrm{~s}$ each so as to gather sufficient information on sessions of different sizes.

\subsection{RED configuration and parameters}

We shall consider four variants of RED and compare them to a drop-tail buffer: the standard RED [4], the adaptive RED [5], and each of these with and without the "gentle" option of RED. The configurations are as follows:

(i) Standard RED: We have used the automatic configuration option of ns (that is set by default). This gave the following values: $\max _{\mathrm{th}}=15, \quad \min _{\mathrm{th}}=5, \quad w_{q}=0.0004$ and $\max _{p}=0.1 .^{4}$

\footnotetext{
${ }^{4}$ Recall that with standard RED, packets start to get rejected when the averaged queue size avg exceeds $\min _{\text {th }}$, and the loss probability increases linearly from 0 to $\max _{p}$ as the average queue size increases between $\min _{\text {th }}$ and $\max _{\text {th }}$. When it exceeds $\max _{\text {th }}$ the dropping probability is one. The avg parameter is initially set to zero. Then with each arriving packet, avg is updated to the value $\left(1-w_{q}\right) \operatorname{avg}+w_{q} q$ where $q$ is the actual queue size and $w_{q}$ is some small constant.
}

(ii) Adaptive RED: [5] The value of the parameter $\max _{p}$ is adapted so as to keep the average queue size within a target range of half way between $\min _{\text {th }}$ and $\max _{\text {th }}$, and it is constrained to the range of $[0.01,0.5]$. The adaptation is done using an additive increase multiplicative decrease policy with parameters $\alpha$ and $\beta$, respectively. We use the default ns parameters of adaptive RED (in particular, we have $\alpha=0.01$ and $\beta=0.9)$.

(iii) Gentle RED: In the gentle RED option, once the averaged queue size exceeds $\max _{\mathrm{th}}$, the drop probability does not jump to 1 , but increases linearly ("gently") to 1 as the average queue size increases to twice $\max _{\mathrm{th}}$.

All our simulations use the same common arrival process of sessions as well as the same size of files (same seeds and generators) so that we can compare easier the effects of the RED version and bandwidth.

\subsection{On the reliability of the simulations}

In Figs. 2 and 3 we present the number of sessions that have been generated during the simulation time. The figures correspond to the gentle RED version with bottleneck link of $1.8 \mathrm{Mbps}$, but it is almost the same in all other variants of RED we simulated, as well as for all other bandwidths (in fact, for larger bottleneck bandwidth we obtain even more sessions of each type) since the figures

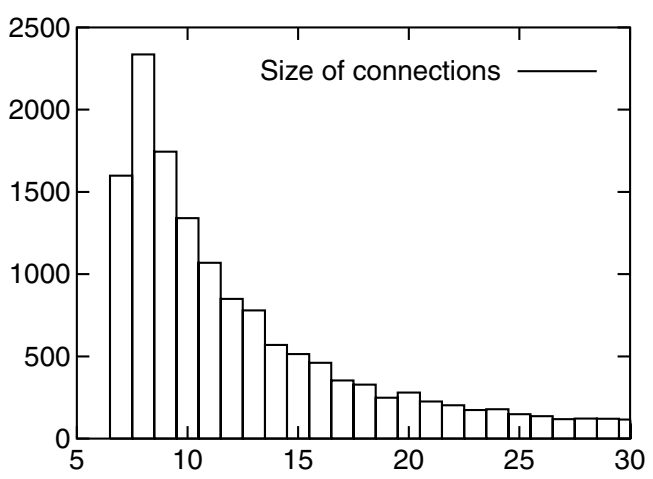

Fig. 2. Number of simulated files ( $y$-axis) as a function of their sizes ( $x$-axis) for files smaller than 30 packets. 


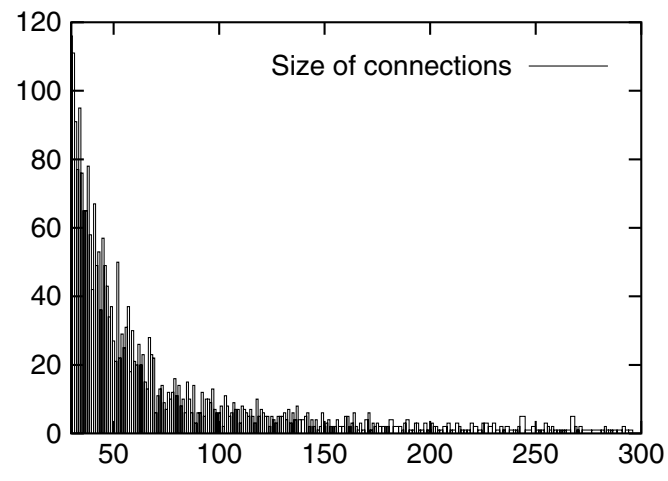

Fig. 3. Number of simulated files ( $y$-axis) as a function of their sizes ( $x$-axis) for files larger than 30 packets.

correspond only to sessions that have terminated, and with larger bandwidth more sessions can terminate.

We observe that up to the size of 100 we get sufficiently many "samples" for each file size (around 10 or more for files of size larger than 30, and 100 or more for files of size less than 30 ). In our statistical analysis we shall also consider files of size larger than 100 . To get sufficiently many samples for that case, we shall aggregate files of neighboring size in our performance analysis. By aggregating 15 neighboring sizes, we are able to get meaningful information up-to files of size 200 (where we get more than 20 files for each group of 15 consecutive sizes). Note that a file of size 200 packets corresponds to 100 kbytes without the header and 108 kbytes with the header. By considering the loss and throughput characteristics of files of sizes of up to 200 packets, we characterize $98.1 \%$ of the file transfers for the Pareto distributed file sizes we have considered. (In fact we present some figures for larger file sizes but the data collected may not be sufficient.)

\section{Preliminary simulation results}

We present in this section some preliminary general simulation results not immediately related to the fairness, that exhibit some strange behavior. We shall understand this behavior when we shall analyze the fairness behavior.

\subsection{Number of sessions}

We depict in Figs. 4 and 5 the total number of active sessions when using the gentle RED version, with the two extreme loading conditions: the "light" load obtained with the bottleneck capacity of $2.5 \mathrm{Mbps}$, and the "heavy" load obtained with 1.8 Mbps. A similar behavior is obtained in all versions of RED we analyzed. Two different time periods are considered.

In the time duration 735-755 s, we observe a strange behavior in which there are from time to time more sessions in the light load regime than in the heavy load regime. This is not generally the case: in the time period $500-525 \mathrm{~s}$ the behavior is exactly the opposite. Although we obtain both

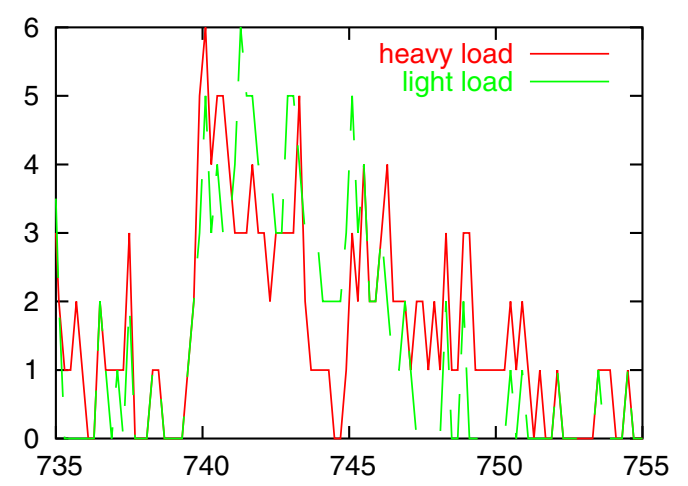

Fig. 4. Active number of sessions ( $y$-axis) as a function of time ( $x$-axis), gentle RED.

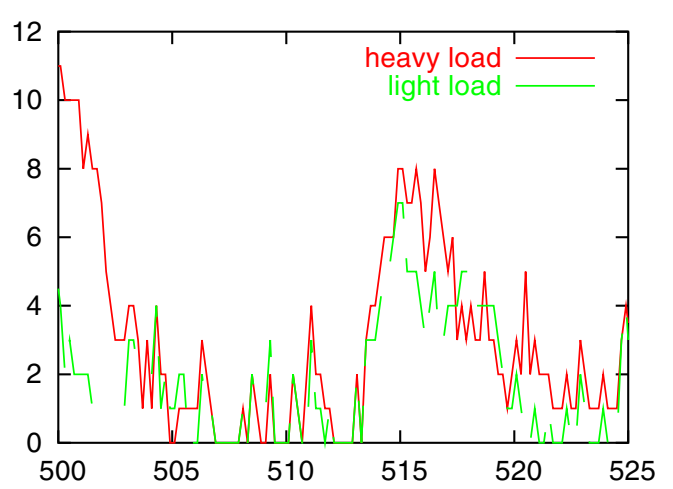

Fig. 5. Active number of sessions ( $y$-axis) as a function of time ( $x$-axis), gentle RED. 
Table 1

Average number of active sessions for various variants of RED and for drop tail as a function of the bottleneck bandwidth

\begin{tabular}{lll}
\hline & $2.5 \mathrm{Mbps}$ & $1.8 \mathrm{Mbps}$ \\
\hline Standard RED & 4.157 & 7.887 \\
Gentle RED & 3.916 & 6.799 \\
Adaptive RED & 4.206 & 6.906 \\
Adaptive gentle RED & 3.999 & 6.906 \\
Drop tail & 6.65234 & 8.323 \\
\hline
\end{tabular}

behaviors, the dominating behavior is the latter as we can see from the Table 1 that presents the average number of active connections. The average number of connection is seen to be the lowest with gentle RED under both high and low loading, and is highest with drop-tail in both cases. The average number of connections for high load (bandwidth of $1.8 \mathrm{Mbps}$ ) is almost double than for the lower load (2.5 Mbps of bandwidth).

\section{Fairness in loss probabilities}

In Figs. 6-8 we present the loss rate of packets as a function of the size of the transfer for various loads corresponding to a bottleneck link of 2.5, 2 and $1.8 \mathrm{Mbps}$, respectively. We actually take a smoother (averaged) version of the loss probabilities where we average over the size of 15 consecutive values of sizes of transfer. Fig. 9 shows an example for the relation between the actual and

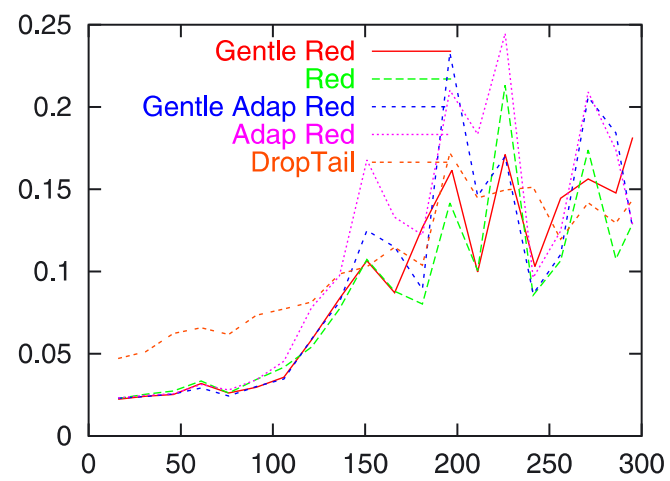

Fig. 6. Loss probability ( $y$-axis) as a function of the transfer size ( $x$-axis) for light load: bottleneck link of $2.5 \mathrm{Mbps}$.

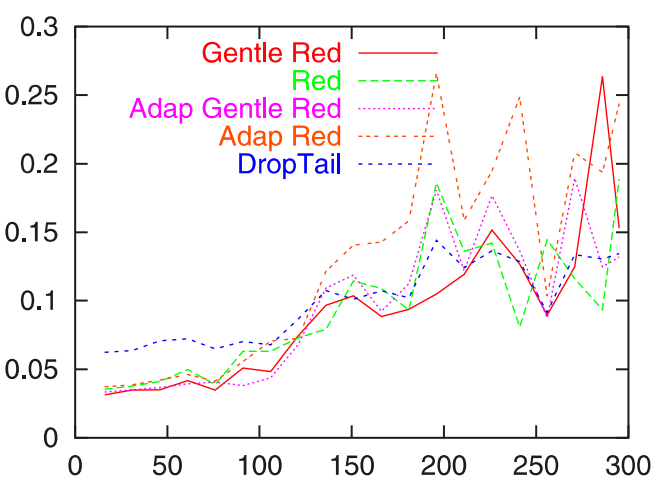

Fig. 7. Loss probability ( $y$-axis) as a function of the transfer size ( $x$-axis) for medium load: bottleneck link of 2 Mbps.

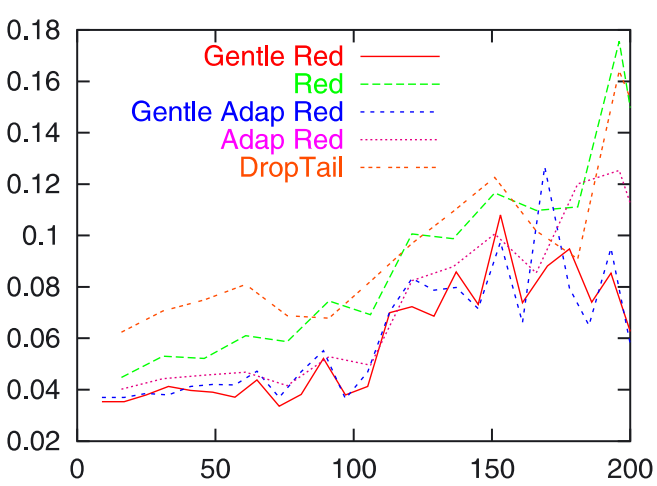

Fig. 8. Loss probability ( $y$-axis) as a function of the transfer size ( $x$-axis) for heavy load: bottleneck link of $1.8 \mathrm{Mbps}$.

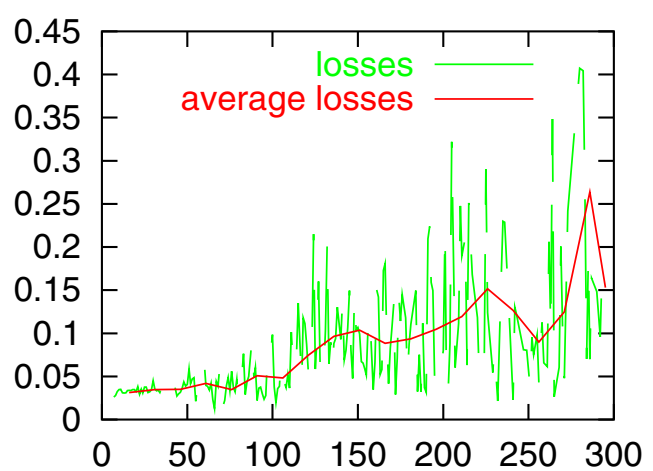

Fig. 9. Exact and averaged loss probability ( $y$-axis) as a function of the transfer size ( $x$-axis) for medium load.

averaged sizes for the case of gentle RED with a bandwidth of the bottleneck link of 2 Mbps. 
In all three Figs. 6-8, drop tail is seen to be the fairest, but this is due in general to a larger amount of drops always observed up to transfer sizes of 100 packets (in heavy losses this is observed for all transfer sizes).

The most unfair results of RED correspond to light load, and the larger the load is, the fairer RED becomes.

In the lightest load case, all versions of RED have an almost constant loss rate of around $2 \%$ for transfer sizes of up to 75 packets long. Then as the size of the transfer increases, the loss rate grows (almost linearly) till it reaches a value of between $15 \%$ and $21 \%$ (depending on the version of RED) of losses for transfers of size 200 packets. Thus an increase in loss rates of a factor of between 7 and 10 is observed as the transfer sizes increase. This shows that the common belief [4, Section IX] that RED is fair with respect to transfer sizes is inaccurate. In our simple bottleneck setting, RED has a bias against long transfers!

In the medium load case, the same behavior is observed but the difference between short and long transfers diminishes. For all versions of RED, we have between $3.3 \%$ and $4 \%$ of loss rates up to transfers of 100 packets long. Then the loss rates increase almost linearly up to values ranging between $11 \%$ and $19 \%$ (depending on the version) for transfers of size 200. We thus obtain a degradation factor of between 3.3 and 6 only.

Finally in the high load, the best fairness is obtained. Drop probabilities are around 4\% for files of sizes up to 70 , and then they grow to values between $10 \%$ and $16 \%$, depending on the version of RED. Thus although the loss rate has a tendency to increase with the size of the transfer, the loss rate is smaller at large file sizes than in the case of light load; at the same time it is larger for small transfer than in light load. The degradation factor we obtain is now between 2.5 and 4 only, which thus exhibits the fairest behavior.

In all three scenarios, gentle RED is both the fairest as well as the most efficient with the loss rates lowest or close to lowest everywhere. In the lightest load, gentle adaptive RED performs better for large transfers.

In Section 6 we shall study the above behavior and find its reasons.
We provide below some more simulation results in order to show the behavior for other parameters as considered so far. In particular, we consider dependence in RTT and also consider lighter load than before.

In Figs. 10 and 11 we show the dependence of the loss rates (as a function of the file transfer) in different values of RTT (the RTT, not including the queueing time, is varied between 4 and 120 $\mathrm{ms}$ ), for RED with the gentle version and for adaptive RED, respectively. We can see that both versions of RED are fairer as the RTTs increase.

We show in Figs. 12 and 13 the dependence of the loss rate (as a function of the size of the transfer) in the load for the Gentle and the adap-

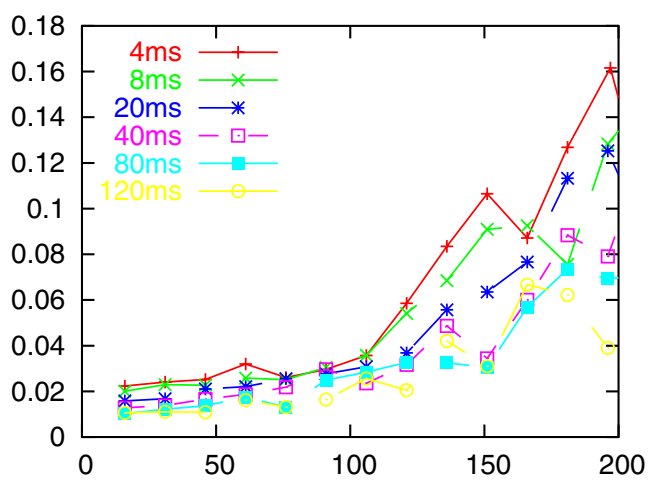

Fig. 10. Loss probability (y-axis) as a function of the transfer size ( $x$-axis) for light load: bottleneck link of $2.5 \mathrm{Mbps}$, for different RTTs and gentle RED.

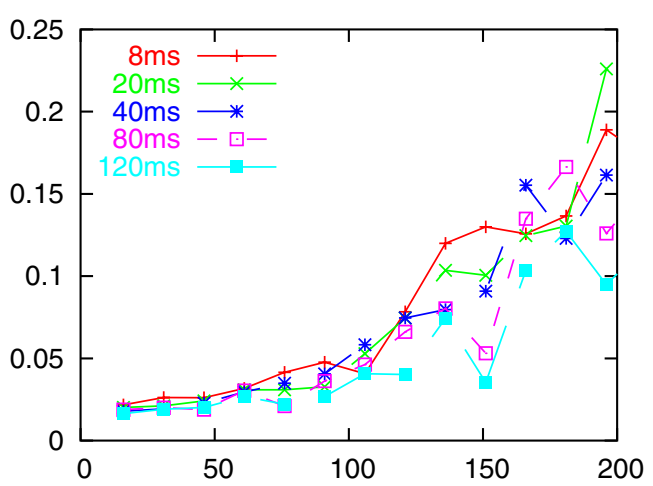

Fig. 11. Loss probability ( $y$-axis) as a function of the transfer size ( $x$-axis) for light load: bottleneck link of $2.5 \mathrm{Mbps}$, for different RTTs and adaptive RED. 


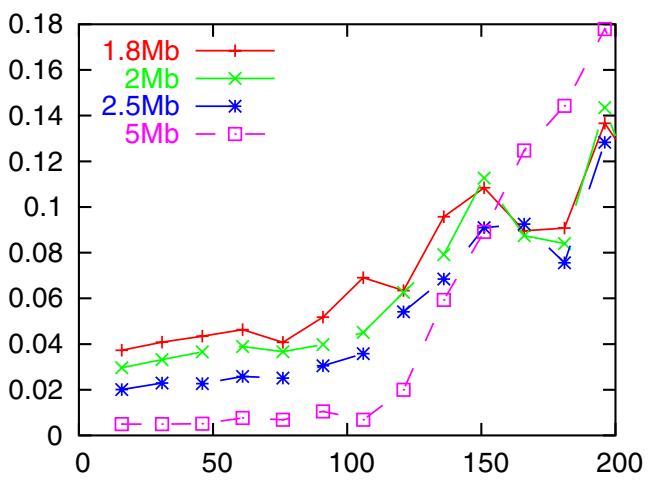

Fig. 12. Loss probability ( $y$-axis) as a function of the transfer size ( $x$-axis) for different loads, gentle RED.

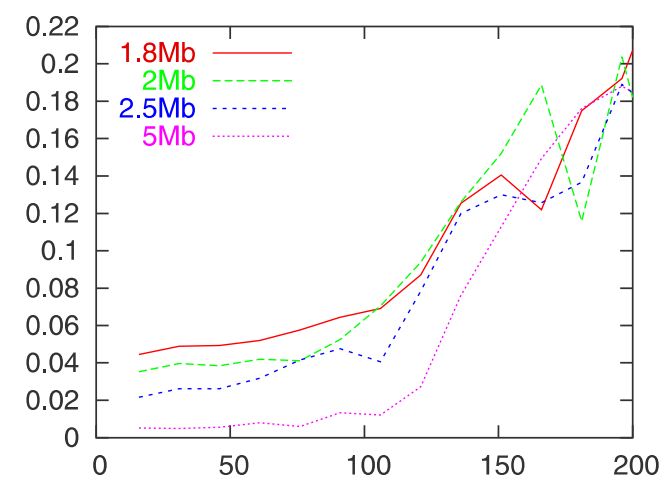

Fig. 13. Loss probability ( $y$-axis) as a function of the transfer size ( $x$-axis) for different loads, adaptive RED.

tive Red versions, respectively. The figure consider bottleneck link capacities varying between 1.8 Mbps $(\rho=0.96)$ and $5 \operatorname{Mbps}(\rho=0.345$ for RTT of $8 \mathrm{~ms}$ (not including queueing). We can see that when there is less load RED is much more unfair. The unfairness obtained at the lightest load in terms of the transfer size is striking: transfer sizes up to around 100 packets suffer almost a constant loss rate of around $0.5 \%$, and then the loss rate increases almost linearly to $18 \%$ for transfer sizes of 200 .

\section{Fairness in throughput}

We repeat the three sets of experiments we did for the loss rates. This time we focus on the fair- ness in the throughput as a function of the size of the transfer for various loads: Figs. 14-16 corresponding to a bottleneck link capacity of 2.5, 2 and $1.8 \mathrm{Mbps}$, respectively. As before, we actually take a smoother (averaged) version of the throughput where we average over the size of 15 consecutive values of sizes of transfer. The relation between the averaged and the non-averaged versions of the throughputs for gentle RED is presented in Fig. 17 for the gentle RED with a bandwidth of the bottleneck link of 2 Mbps.

Our first observations from the curves are (i) For all transfer sizes, any of the RED versions outperforms the throughput of the drop-tail buffer; (ii) The performances of the various variants of RED are very similar; (iii) The throughputs are decreasing as the load increases (i.e. as the bottleneck link

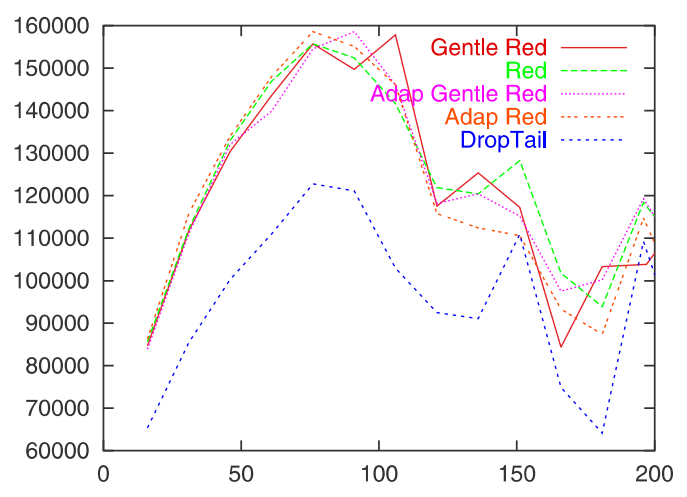

Fig. 14. Throughput (y-axis) as a function of the transfer size (x-axis) for light load: bottleneck link of 2.5 Mbps.

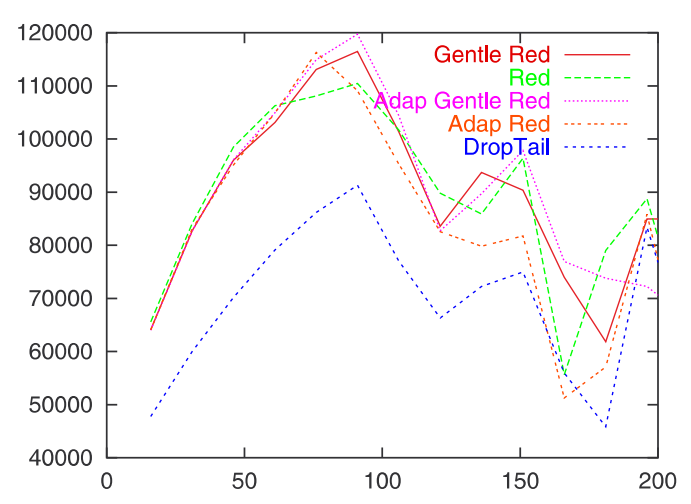

Fig. 15. Throughput ( $y$-axis) as a function of the transfer size ( $x$-axis) for medium load: bottleneck link of 2 Mbps. 


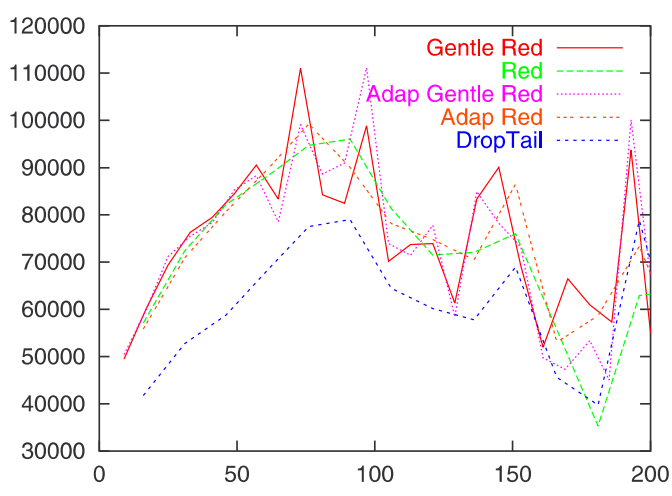

Fig. 16. Throughput ( $y$-axis) as a function of the transfer size ( $x$-axis) for heavy load: bottleneck link of $1.8 \mathrm{Mbps}$.

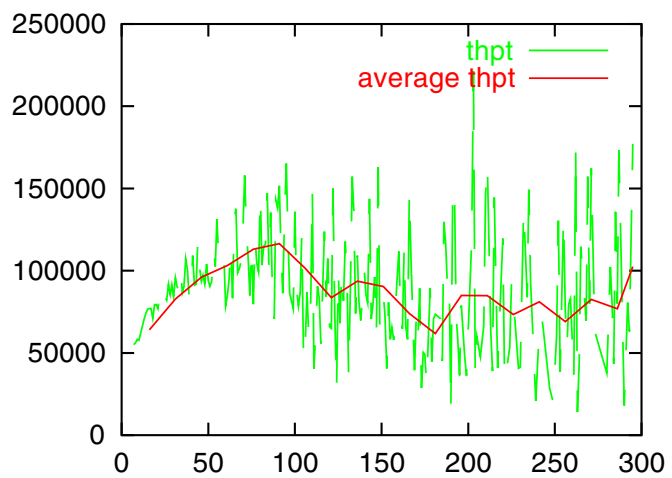

Fig. 17. Exact and averaged throughput (y-axis) as a function of the transfer size ( $x$-axis) for medium load.

capacity decreases); (iv) Unlike the loss probabilities that tended to increase with the transfer size, the throughput first increases, then decreases again. The increase coincides approximately with the region in which the loss probabilities were constant, and is due to the fact that larger window sizes are achieved when the transfers are larger. Then the decrease of the throughput is related to the increase in the loss probabilities that we observed previously.

As for fairness in the throughput as a function of the transfer size, we observe the following: (i) The throughput as a function of the transfer size has the same form for all loads. The maximum throughput is in all three cases around twice the minimum throughput; (ii) Throughput is thus much more fairly distributed as a function of transfer sizes than the loss probabilities; (iii) All variants of RED seem slightly fairer than the drop tail.

In Figs. 18 and 19 we show the variability in throughput when changing the RTT for gentle RED and adaptive RED, respectively. The throughput itself is seen, as expected, to decrease with RTT, but the amount of "unfairness" in the throughput does not seem to be much influenced by RTT.

We show in Figs. 20 and 21 the dependence of the throughput (as a function of the size of the transfer) in the load for the Gentle and the adaptive Red versions, respectively. We varied the bottleneck link capacities between $1.8 \mathrm{Mbps}$

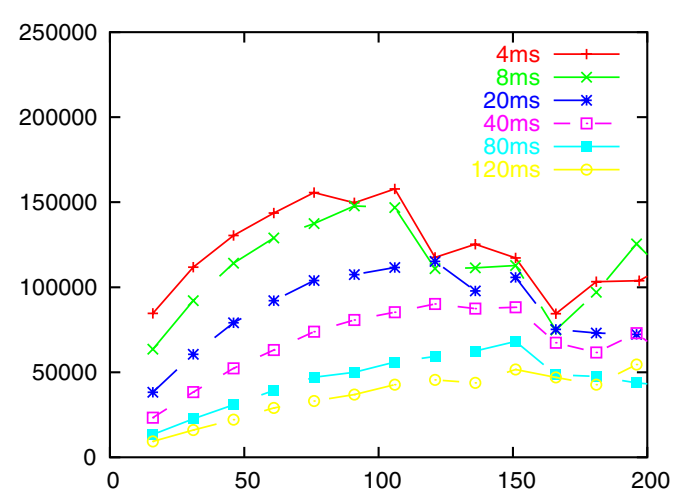

Fig. 18. Throughput ( $y$-axis) as a function of the transfer size ( $x$-axis) for light load: bottleneck link of $2.5 \mathrm{Mbps}$ and different RTTs.

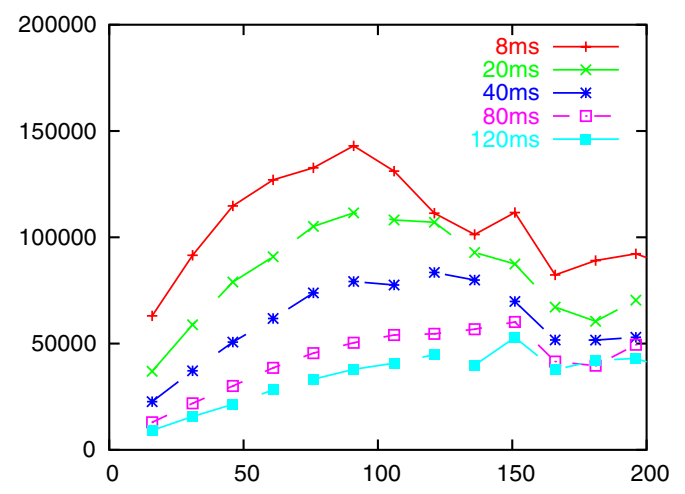

Fig. 19. Throughput (y-axis) as a function of the transfer size ( $x$-axis) for light load: bottleneck link of $2.5 \mathrm{Mbps}$ and different RTTs. 


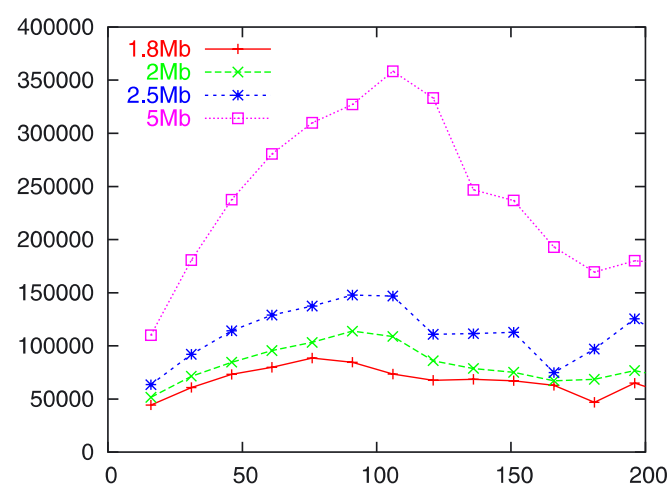

Fig. 20. Throughput ( $y$-axis) as a function of the transfer size ( $x$-axis) for different loads, gentle RED.

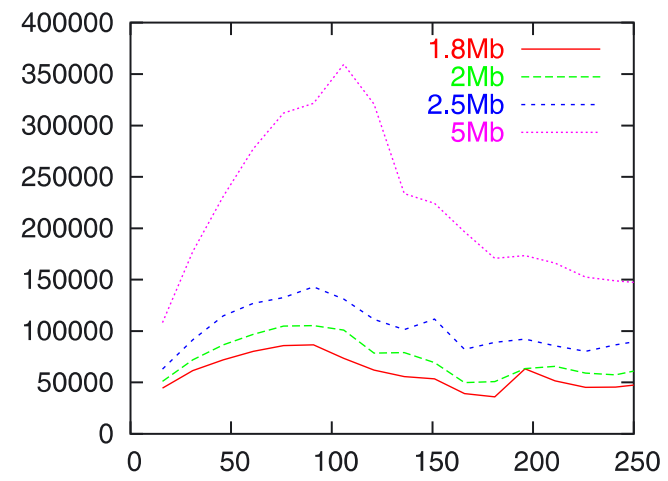

Fig. 21. Throughput ( $y$-axis) as a function of the transfer size ( $x$-axis) for different loads, adaptive RED.

( $\rho=0.96)$ and 5 Mbps $(\rho=0.345)$ for RTT of 8 $\mathrm{ms}$ (not including queueing). We can see that when there is less load RED is much more unfair. For the lightest load (obtained with a bottleneck link of $5 \mathrm{Mbps}$ ) the largest throughput, obtained for files of sizes of around 100 packets, is around 3.5 twice larger than the throughput of files with lowest size, where as for the largest load (obtained with a bottleneck link of $1.8 \mathrm{Mbps}$ ), the difference is less than twice.

\section{Analysis of the fairness behavior}

In order to understand the "bias" against long transfers, we focus on some arbitrarily chosen long transfer that suffered many losses. We chose a transfer of 155 packets, that involved a total number of 201 transmitted packets (including retransmissions) when using gentle RED with the light load (bottleneck link of $2.5 \mathrm{Mbps}$ ). When using $1.8 \mathrm{Mbps}$, the same transfer required only a total of 171 transmissions plus retransmissions.

We first present in Figs. 22 and 23 the evolution of sequence number of transmitted packets as a function of time in both cases, and in Fig. 24 we present the evolution of window size for both cases. We see that in the light load case there is a large initial burst; it involves many losses since many packets are seen to be later retransmitted. The transmission in the case of heavy load does not start in a bursty way, drops occur earlier which

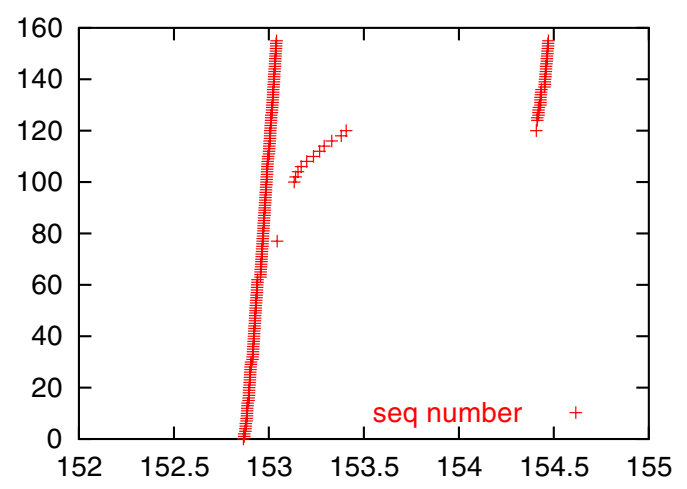

Fig. 22. Sequence number ( $y$-axis) of transmitted packets for gentle RED as a function of time ( $x$-axis), bottleneck link of 2.5 Mbps.

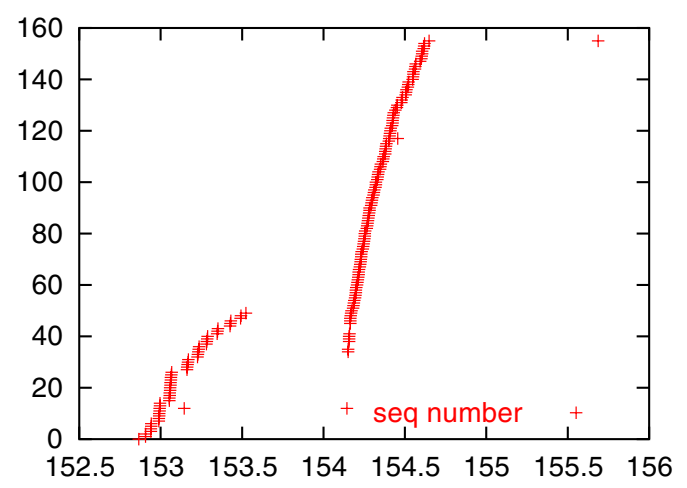

Fig. 23. Sequence number ( $y$-axis) of transmitted packets for gentle RED as a function of time ( $x$-axis), bottleneck link of 1.8 Mbps. 


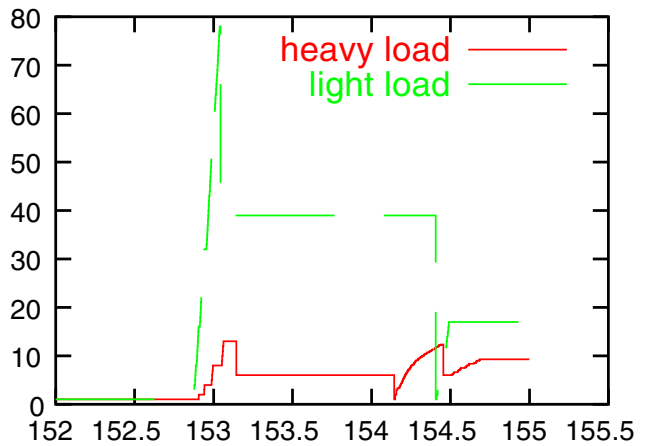

Fig. 24. Window size evolution (y-axis) of a connection that suffered many losses for gentle RED in light load as a function of time ( $x$-axis).

smoothes the traffic. This smoother increase in window size due to earlier losses finally results in less losses in the overall transfer. To understand this better, we plot the evolution of the queue size and RED's average queue size for both cases in Figs. 25 and 26. We see that out transfer caused a peak in the instantaneous queue size at time $153 \mathrm{~s}$ approximately that resulted in a queue overflow; this occurred in both cases. However, we see that the average queue size is larger for the heavy load case, which resulted in drops occurring earlier due to RED early discarding. This explains the more smooth behavior of the transmission at heavy load, and thus its better performance in terms of losses. This type of phenomenon can also explain

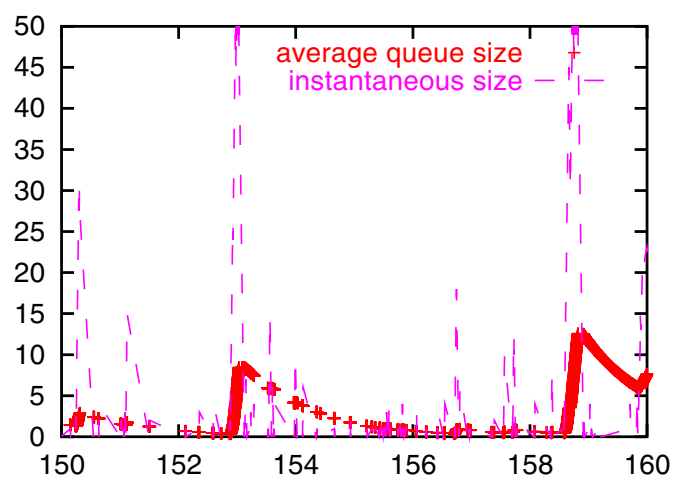

Fig. 25. Average and instantaneous queue size ( $y$-axis) for gentle RED as a function of time ( $x$-axis), bottleneck link of 2.5 Mbps.

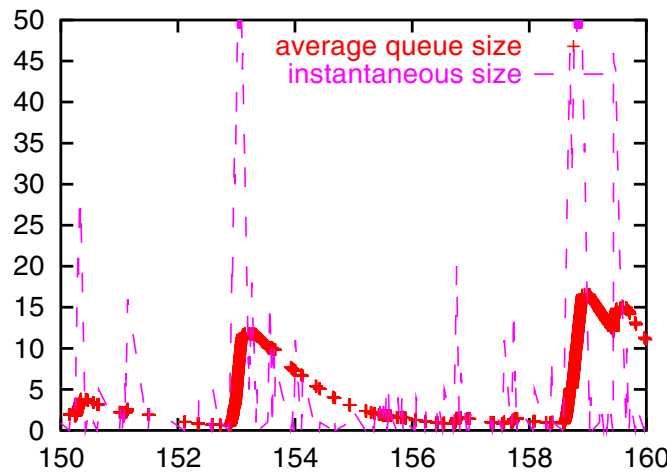

Fig. 26. Average and instantaneous queue size (y-axis) for gentle RED as a function of time ( $x$-axis), bottleneck link of 2.5 Mbps.

the strange behavior we observed in Section 3, where in some cases, the number of active sessions was larger for the lighter load than for the larger one.

\section{Conclusions}

We examined how loss rate of a transfer and the transfer throughput are related to its size. We have seen that RED is biased against long connections in terms of loss probabilities but that this bias decreases as the load increases. In terms of throughput, RED is fairer than in terms of the loss rate. Best performance has been obtained with gentle RED, and in general all variants of RED performed better than drop-tail.

In our simulation scenarios, file transfers could be identified with bursts. For example, "spikes" in Figs. 25 and 26 correspond to file transfers. In that case, fairness in terms of the size of a file can be identified with fairness in terms of burst size. Our conclusions on fairness in terms of burst size are then different than those that had been obtained in previous work through simulations of permanent connections.

\section{References}

[1] A. Arvidsson, P. Karlsson, On traffic models for TCP/IP, in: Proceedings of 16th International Teletraffic Congress (ITC16), Edinburgh, UK, June 1999. 
[2] T. Bonald, M. May, J. Bolot, Analytic evaluation of RED performance, IEEE Infocom 2000.

[3] B. Braden et al., Recommendations on queue management and congestion avoidance in the Internet, RFC 2309, April 1998.

[4] S. Floyd, V. Jacobson, Random early detection gateways for congestion avoidance, IEEE/ACM Transactions on Networking 1 (4) (1993) 25-39.

[5] S. Floyd, R. Gummadi, S. Shenker, Adaptive Red: an algorithm for increasing the robustness of RED's active queue management, unpublished, 2001.

[6] C.V. Hollot, V. Misra, W.-B. Gong, On designing improved controllers for AQM routers supporting TCP flows, in: Proceedings of INFOCOM 2001.

[7] Y. Joo, V. Ribeiro, A. Feldmann, A.C. Gilbert, W. Willinger, TCP/IP traffic dynamics and network performance: a lesson in workload modeling, flow control, and trace-driven simulations, ACM/SIGCOMM Computer Communication Review 31 (2) (2001).

[8] A. Kantawala, J. Turner, Queue management for shortlived TCP flows in backbone routers, in: High Speed Symposium, IEEE Globecom, Taipei, Taiwan, 2002.

[9] P. Kuusela, P. Lassila, J. Virtamo, P. Key, Modeling RED with idealized TCP sources, in: Proceedings of 9th IFIP Working Conference on Performance Modeling and Evaluation of ATM and IP Networks, June 2001.

[10] D. Lin, R. Morris, Dynamics of random early detection, Sigcomm, Cannes, France, October 1997, pp. 127-137.

[11] S.H. Low, F. Paganini, J. Wang, S. Adlakha, Dynamics of TCP/RED and a Scalable Control, in: Proceedings of INFOCOM, New York, USA, June 2002.

[12] V. Misra, W. Gong, D. Towsley, Fluid-based analysis of a network of AQM routers supporting TCP flows with an application to RED, in: Proceedings of ACM Sigcomm, Stockholm, Sweden, 2000.

[13] H. Ohsaki, M. Murata, H. Miyahara, Steady state analysis of the RED gateway: Stability, transient behavior, and parameter setting, IEICE Transactions on Communications 85 (1) (2002) 107.

[14] T.J. Ott, T.V. Lakshman and L. Wong, SRED: Stabilized RED, Proceedings of Infocom, vol. 3, 1999, pp. 1346-1355.
[15] V. Sharma, P. Purkayastha, Performance analysis of TCP connections with RED control and exogenous traffic, in: Proceedings of IEEE Globecom, 2001.

[16] P. Tinnakornsrisuphap, A.M. Makowski, Queue dynamics of RED gateways under large number of TCP flows, Globecom, San Antonio, TX, USA, November 2001.

[17] T. Ziegler, C. Brandauer, S. Fdida, A quantitative model of RED with TCP traffic, in: Proceedings of IEEE/ACM/IFIP IWQoS, Karlsruhe, Germany, June 2001.

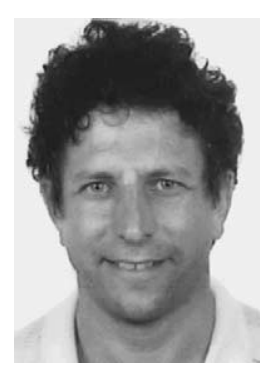

Eitan Altman received the B.Sc. degree in Electrical Engineering (1984), the B.A. degree in Physics (1984) and the Ph.D. degree in Electrical Engineering (1990), all from the Technion-Israel Institute, Haifa. In 1990, he further received his B.Mus. degree in Music Composition in Tel-Aviv University. Since 1990, he has been with INRIA (National Research Institute in Informatics and Control) in Sophia-Antipolis, France. His current research interests include performance evaluation and control of telecommunication networks and in particular congestion control, wireless communications and networking games. $\mathrm{He}$ is in the editorial board of several scientific journals: Stochastic Models, JEDC, COMNET, SIAM SICON and WINET. He has been the (co)chairman of the program committee of several international conferences and workshops (on game theory, networking games and mobile networks). More informaion can be found at http:// www.inria.fr/mistral/personnel/Eitan.Altman/me.html.

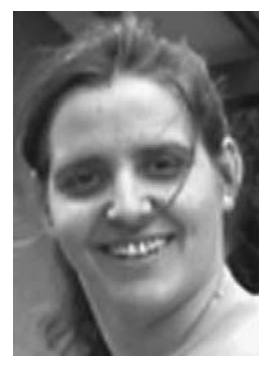

Tania Jiménez received her DEA (equivalent to M.Sc) at 1997, and Ph.D. at 2000, both in University of Nice Sophia-Antipolis, in networks and distributed systems. Her research interests include simulation as well as optimization and control of telecommunication networks. She has been a teaching and research assistant at Nice University, teaching computer science courses. She is now a lecturer at CESIMO, Facultad de Ingenieria, Univ. de Los Andes, Merida, Venezuela. 